\title{
Estrategias de comunicación de las editoriales literarias de España
}

\author{
Almudena Gómez López* \\ Pedro Antonio Hellín Ortuño*
}

Artículo recibido:

21 de junio de 2018

Artículo aceptado:

22 de enero de 2019

Artículo de investigación

\section{Resumen}

Este estudio trata el tema del marketing editorial y las estrategias de comunicación que despliegan las editoriales literarias en España, y tiene como objetivos fundamentales servir de base a otros estudios referentes a la misma área, conocer las estrategias de comunicación de algunas editoriales literarias de España y establecer una delimitación conceptual del marketing editorial. La metodología empleada consta de una investigación documental en bases de datos, páginas web y bibliotecas, que ha servido para recoger, seleccionar y analizar la información más importante sobre el tema tratado, así como una investigación de campo mediante entrevistas a los responsables del área de mercadotecnia de las editoriales Alba, Anagrama y Balduque para conocer el uso que hacen de las estrategias de comunicación.

* Facultad de Comunicación y Documentación, Universidad de Murcia, España almudena.gomez@um.es phellin@um.es

INVESTIGACIÓN BIBLIOTECOLÓGICA, vol. 33, núm. 80, julio/septiembre, 2019, México, ISSN: 2448-8321 pp. 57-71 
Los resultados obtenidos evidencian un uso progresivo de la comunicación digital como medio de información, publicidad y fidelización de lectores, con una mejor adaptación a las redes sociales de una editorial pequeña, pero con mayor uso de sus herramientas de publicidad de una editorial grande, siendo similares el uso de las estrategias de comunicación convencionales. Las conclusiones ponen de manifiesto la necesidad de conceptualizar el marketing editorial, debido a la confusión de su utilización, las estrategias de comunicación poco definidas y un uso analógico de las nuevas tecnologías.

Palabras clave: Marketing Editorial; Estrategias de Comunicación; Editoriales Literarias.

Communication strategies of literary publishing houses in Spain

Almudena Gómez López and Pedro Antonio Hellín

Ortuño

\section{Abstract}

The study deals with the topic of editorial marketing and communication strategies carried out by literary publishing houses in Spain, and its main objectives are to serve as a basis for other studies related to the same area, to know the communication strategies of some literary publishing houses in Spain and establish a conceptual delimitation of editorial marketing. The methodology used consists of a documentary research in databases, webs and libraries that have served to gather, select and analyze the most important information on the subject, and a field research through interviews with the marketing managers of publishing houses Alba, Anagrama and Balduque to know the use they make of communication strategies. The results obtained show a progressive use of digital communication as a means of information, advertising and loyalty of readers, with a better adaptation to the social media of a small publishing house but with greater use of its advertising tools of a large publishing house, being similar the use of conventional communication strategies use. The conclusions highlight the need to conceptualize editorial marketing due to the confusion of its use, little defined communication strategies and an analogical use of new technologies.

Key Words: Editorial Marketing; Communication Strategies; Literary Publishing Houses. 


\section{INTRODUCCIÓN}

T a realidad española actual del marketing editorial como especialización

Ldentro de la mercadotecnia presenta una discontinuidad en lo referente a su estudio y aplicación de sus técnicas, así como la necesidad de establecer unos procedimientos de actuación en lo relativo a las investigaciones realizadas respecto de las editoriales y sus estrategias, el producto-libro y a los consumidores de éste como bien cultural.

El motivo que ha llevado a la realización de este estudio se basa en la escasez de investigaciones que abordan el tema del marketing editorial en general y las estrategias de comunicación de las editoriales en concreto.

La información existente sobre la empresa editorial se basa en la realización de informes anuales o bianuales de la situación del sector editorial y los hábitos de lectura en España, mientras que las investigaciones referentes a la comunicación editorial se engloban dentro de los escasos estudios que hay sobre marketing editorial en España y todos ellos con referencia en el libro Marketing editorial: cómo satisfacer las necesidades de los lectores de libros de Daniel Gómez-Tarragona (2010).

El presente estudio aborda la cuestión de las estrategias de comunicación de las editoriales literarias de España, y su importancia se basa en tres motivos principales: la unificación de la información más importante que hay sobre el sector editorial y la comunicación editorial en España, que es muy escasa y hasta ahora se encontraba dispersa, lo que nos lleva a la segunda razón, y es que al concentrar la información sobre el tema en cuestión, el estudio sirve como punto de partida a otras líneas de investigación relacionadas con el marketing y la comunicación editorial. El último motivo es que aporta información sobre las estrategias de comunicación de algunas editoriales literarias de España, lo que da pie a una continuidad del estudio.

Así pues, esta investigación tiene como objetivo general servir de base a otros estudios relacionados con las estrategias de comunicación de las editoriales literarias de España, teniendo como objetivos más específicos el conocimiento del uso que se hace de las estrategias de comunicación en algunas editoriales literarias españolas y el establecimiento de una delimitación conceptual de la mercadotecnia editorial. 


\section{Aproximación al concepto de marketing editorial}

El marketing (o mercadotecnia) editorial como concepto no está estandarizado en España, por lo que las editoriales no tienen una representación nominal unitaria que defina sus acciones para con la empresa y sus consumidores. Tan sólo cuenta con aproximaciones al concepto como el siguiente:

Una combinación de términos que más de uno juzgará contradictoria. El sustantivo alude a una serie de prácticas estandarizadas y en apariencia mecánicas que no casan con el adjetivo, en el que se condensa la actividad fecunda y de alguna manera siempre nueva de quien publica libros. Más aún, a menudo el maridaje entre los principios mercadológicos y los de la creatividad editorial suelen terminar en insanas relaciones de pareja, en la que se imponen los dictados de una parte a costa de la otra —y no está claro cuál de los dos desequilibrios es más perjudicial para los lectores-(Cole, 2003: 13).

Asimismo, Gómez-Tarragona (2010: 15) no conceptualiza el marketing editorial en una definición concreta, sino que hace hincapié en la importancia que tiene el empleo de las técnicas del mercadeo para un editor como transmisor de la cultura, ya que le permite conocer y comprender las necesidades de sus consumidores, y alcanzar así sus objetivos empresariales y culturales.

\section{El mercado del libro en España: panorama actual}

La empresa editorial se halla en una situación actual de cambios constantes, debido a factores como el incremento de publicaciones, la aparición de grandes superficies que las distribuyen o el desarrollo de la Internet, entre otros. En los últimos años, ha aumentado el precio medio del libro: ascienden las publicaciones, pero desciende la venta de ejemplares y se multiplican los centros de distribución del producto, siendo las librerías las que obtienen un mayor porcentaje de ventas. Respecto del libro como bien cultural, el libro impreso sigue siendo el principal producto de las editoriales frente al libro electrónico, que aumenta su lectura y venta. La crisis económica ha afectado a la industria editorial española, descendiendo las ventas y aumentando la lectura en bibliotecas, por lo que las editoriales se ven obligadas a desarrollar estrategias que compensen el aumento de precios de los libros, como los descuentos o las ventas de ocasión. 
Respecto de esta información, los informes del sector del libro más actualizados aportan los siguientes datos. En 2015, se contabilizan en España 2,963 editoriales en activo, 134 menos que en el año anterior. En 2016, el número de editoriales en activo ascendió a 3,026, aumentando el número de editoriales independientes.

La edición aumentó en 2015, alcanzando los 79,397 títulos, un 0.2 por ciento más que en 2014; mientras que en 2016 ascendió considerablemente en un 8.3 por ciento con 86,000 títulos. La edición en papel aumenta y representa cerca del 72 por ciento de la edición española, pero el libro en soporte digital ya representa el 27.5 por ciento de la edición en 2016, aumentando en los últimos años.

Madrid y Cataluña condensan más del 62 por ciento de la edición nacional.

Respecto del total editado, el libro en lengua española representa el 91.4 por ciento en 2016 (92.9 por ciento en 2015 y 91.7 por ciento en 2014) y en lengua extranjera el 6.7 por ciento (5.1 por ciento en 2015 y 5.9 por ciento en 2014).

En 2014 inició un proceso de recuperación del sector editorial, el cual se consolidó en 2016 con la venta de 1.2 millones de ejemplares más y un aumento en la facturación respecto de 2015 superior a los sesenta millones de euros.

El precio medio del libro de papel en 2015 fue de 20.93 euros, aumentando considerablemente en los últimos años; mientras que en 2016 descendió hasta los 19.80 euros. El libro digital se mantiene en los 12 euros.

La librería es el principal canal de venta de libros, concentrando aproximadamente el 35 por ciento de la facturación, mientras que las cadenas de librerías concentran el 53 por ciento de las ventas. Una de cada cuatro librerías vende sus libros de forma online, representando el 11 por ciento de sus ventas, y una de cada diez vende libros digitales. Amazon es el principal canal de venta en línea.

La lectura es una de las actividades culturales más practicada por los españoles, con un 62.2 por ciento de personas que afirman haber leído algún libro en 2016. El hábito de lectura crece un 3.5 por ciento en los últimos años.

España es una de las principales potencias editoriales a escala mundial, situándose en sexto lugar por habitantes, por detrás de Alemania, Noruega, Estados Unidos, Reino Unido y Australia, y en noveno puesto por volumen de ventas. El libro de texto y la novela acaparan las principales ventas de las editoriales españolas.

\section{Metodología}

El estudio realizado es cualitativo, de naturaleza exploratoria y se divide en dos fases: con el objetivo de conocer la información existente sobre el tema 
en cuestión, la primera fase consta de una investigación documental en la que se ha procedido a la recopilación de información y su correspondiente tratamiento; la segunda fase consiste en una investigación de campo, con el objetivo de analizar las estrategias de comunicación de algunas editoriales literarias de España, para saber el uso que hacen éstas de las estrategias de comunicación, y encontrar puntos comunes y diferencias entre sí, relacionarlas con las estrategias de comunicación definidas en el artículo y, finalmente, interpretar los resultados y sacar unas conclusiones generales.

\section{Investigación documental}

Búsqueda y recogida de información primaria sobre marketing editorial, comunicación editorial, estrategias de comunicación editorial, el estado de la cuestión en España y otros términos temáticos en el buscador de Google, en Google académico, Google Books y bases de datos como SciELO, Dialnet y Redalyc, en el catálogo colectivo de la Biblioteca Regional de Murcia y en la Biblioteca General de la Universidad de Murcia; lectura y selección de los materiales a utilizar y criba de los innecesarios por carecer de representatividad o considerarse repetitivos.

Tratamiento de la información: la elaboración del artículo a partir de la información analizada se realiza de lo general a lo particular, comenzando por una aproximación al concepto de marketing editorial, una revisión del panorama editorial en los últimos años en España y una discusión en la que se concretan y especifican las estrategias de mercadotecnia que se realizan en las editoriales, las cuales sirven para contrastar los resultados que se obtienen en la investigación de campo.

\section{Investigación de campo}

Análisis de estrategias y resultados: la metodología empleada para el análisis de las estrategias de comunicación de algunas editoriales literarias de España se basa en una investigación cualitativa, mediante la realización de entrevistas a través de cuestionarios con preguntas abiertas a los responsables de mercadotecnia de las editoriales escogidas. La entrevista se ha realizado de dos formas: personal y por correo electrónico, y su estructura consta de preguntas introductorias, de contenido y de conclusiones, que van de lo general a lo particular. Elaboración de la lista de editoriales, contacto con éstas y envío 
del cuestionario y realización de la entrevista personal. Esta última se realizó mediante una grabación de voz que posteriormente fue transcrita. Recogidas las entrevistas, se procedió a su análisis, ya sea por preguntas concretas, o por bloques de preguntas que siguen un orden lógico para poder unirlas.

El cuestionario versa sobre el proceso de promoción de los libros; la lista definitiva de editoriales con las que se ha contactado, de forma telefónica o por correo electrónico, es la siguiente:

1) Tirano Banderas

2) Grupo Planeta

3) Balduque

4) Salamandra

5) $\mathrm{SM}$

6) Alfaguara

7) Edebé

8) RBA

9) Alianza

10) Anagrama

11) Santillana

12) Alba

13) McGraw Hill

14) Vision Net

15) Signo

16) Penguin Random House

17) Cátedra

18) Vicens Vives

19) Laberinto

20) Akal

21) Edicions 62

22) Espasa

23) Gigamesh

24) Libros del asteroide

25) Maeva

26) Milenio

27) Minúscula.

Finalmente, las editoriales que contestaron al cuestionario fueron Balduque, mediante una entrevista personal; las editoriales McGraw Hill, Alba y Anagrama, vía correo electrónico. Se decidió descartar la editorial McGraw 
Hill por no ser una editorial especializada en literatura, por lo que no se le considera representativa.

El cuestionario se divide en cinco partes, con una introducción con preguntas sobre el panorama editorial actual y cómo afecta a las editoriales; las tres siguientes versan sobre el proceso de promoción de los libros antes, durante y después de la publicación del libro, respectivamente, y unas conclusiones de la propia editorial entrevistada sobre sus debilidades, fortalezas y oportunidades.

\section{Discusión}

Los estudios sobre marketing editorial no aportan una definición concreta que sintetice sus funciones, por lo que podemos definirlo como el conjunto de técnicas de mercadotencia aplicadas al ámbito editorial, que incluye la creación de un plan de comercialización con objetivos de crecimiento empresarial, rentabilidad y la satisfacción de los consumidores-lectores. Aquí definimos las estrategias de comunicación que aportan un conocimiento general de su desarrollo en las editoriales y nos sirve para saber el uso que se hace de esas estrategias en las editoriales analizadas, así como las similitudes y diferencias entre aquéllas.

\section{Estrategias de mercadotecnia de las editoriales literarias de España}

Según Gómez-Tarragona (2010), las estrategias de marketing están delimitadas por los objetivos que se quieren conseguir, que se establecen tras realizar el análisis interno y externo para conocer la realidad editorial actual. A grandes rasgos, los objetivos de las editoriales grandes y medianas se establecen en términos de crecimiento; mientras que las pequeñas buscan la rentabilidad a corto plazo.

Las estrategias de marketing de una editorial se realizan a través de las herramientas del "marketing mix":

Las estrategias relativas al producto editorial se basan en la creación de marca por parte de la editorial que permita su diferenciación y posicionamiento respecto de la competencia; la construcción de su reputación, a través de la configuración de su catálogo (títulos, autores, etc.), basándose en la satisfacción de los clientes con el producto, la calidad e inversión en comunicación y el desarrollo de los valores 
emocionales. El catálogo debe contener tanto las novedades como los libros del fondo editorial, organizado en colecciones y series, culminando en la creación de un plan editorial con parámetros, como la lista de libros que se van a publicar, títulos, autores, colecciones, mes y semana de lanzamiento, formato, encuadernación y venta estimada, entre otros; y el diseño del libro, que cumple una función comercial y define el estilo de la editorial. Así, las cubiertas son un elemento de gran importancia, tanto si son tipográficas o ilustrativas, ya que su atractivo ayuda a vender; en las contracubiertas se incluye el texto explicativo del libro y testimonios o críticas del mismo. El lomo del libro puede aportar un atractivo añadido, ya que es lo primero que se ve cuando está colocado en una estantería. Las cintas que envuelven al libro tienen una función meramente publicitaria ya que contienen cifras de ejemplares vendidos de ese mismo libro (Gómez-Tarragona, 2010: 81-90).

El precio del libro lo establece el editor, siendo éste un incentivo para la demanda o una estrategia contra la competencia, además de servir para generar beneficio y rentabilidad para la propia empresa. El precio se determina atendiendo a los costes de producción del libro, al valor que los propios clientes le dan al producto, según los precios de la competencia y los aspectos legales, ya que el editor impone un precio fijo, del que sólo se puede hacer un 5 por ciento de descuento o un 10 por ciento en las ferias del libro, así como los objetivos y estrategias de la editorial y la previsión de ventas del libro. Para establecer un precio óptimo de éste, las editoriales buscan el punto medio, en el que los consumidores estén satisfechos con lo que gastan y que resulte rentable para la empresa, por lo que el precio máximo es establecido por los consumidores, pues son ellos quienes determinan lo que están dispuestos a pagar, mientras que el mínimo lo fijan los propios editores.

Las estrategias de distribución y comercialización de libros conllevan un proceso de ventas cuyas fases son la búsqueda e identificación de los clientes potenciales, la presentación del producto, la información adicional, la venta y el servicio posventa. Para el buen desempeño de cada una de estas fases, se desarrollan estrategias como la formación constante del personal de ventas, a través de jornadas en las que se expliquen los servicios de la editorial o fichas de producto en las que se detallan las características de los libros a vender y argumentos de venta, entre otros; descuentos y devoluciones a los distintos agentes involucrados en el proceso de distribución y comercialización del producto; elección de los canales de distribución para la venta del libro, basándose en el análisis del producto, la calidad de los servicios de cada canal y la idoneidad de los objetivos y del producto respecto de los canales existentes (Gómez-Tarragona, 2010: 141-142).

David Cole (2003: 22-23) recomienda que las editoriales sepan a qué nicho de mercado se quieren dirigir y que vigilen los movimientos de la competencia, 
ya que un libro tendrá una clara superioridad sobre otro de la competencia si trata sobre un tema que ha ganado popularidad, si su autor es conocido o si aporta un nuevo enfoque sobre un tema ya explotado.

Para dar a conocer su producto-libro, generar una actitud positiva hacia éste y relacionarse con el mercado, las editoriales disponen de distintas herramientas de comunicación.

Las editoriales pequeñas suelen destinar sus esfuerzos a acciones de comunicación de menor coste económico, como el marketing interactivo, por el que se comunican con los lectores mediante páginas web, redes sociales o la venta en línea de ejemplares. Otras acciones de comunicación que ofrecen son de relaciones públicas y publicidad, como la elaboración de notas de prensa, presentaciones de libros y entrevistas con el autor para los medios de comunicación.

Las grandes editoriales, por el contrario, emplean todas las herramientas de comunicación integral, como las relaciones públicas, además de las ya mencionadas, promocionan a los autores y sus libros en ferias, así como en librerías mediante encuentros con autores o acciones de venta personal, aunado a la venta en línea y la promoción a través de redes sociales o páginas web. Respecto de los clásicos o con autores contemporáneos ya consagrados, suelen emplear acciones de promoción de ventas, como descuentos u ofertas de valor añadido, por el que, además del producto-libro en sí, se pueden obtener extras como regalos u otros artículos.

La herramienta de comunicación más costosa es la publicidad, por lo que medios como la televisión o la radio sólo serán empleados por los grandes grupos editoriales, sólo cuando se trata de autores ya consagrados. Así, los medios más indicados para promocionar libros y autores son las revistas especializadas en literatura, como Qué leer, Quimera, Delibros, o los suplementos culturales de periódicos españoles como Babelia, de El País, ABCD las artes y las letras, de $A B C$, o El Cultural, de El mundo (Escribano, 2012: 14).

\section{Presentación y ANÁlisis DE RESUltados}

El proceso de transformación en el que se encuentran las editoriales tiene como denominador común la crisis económica, el auge de las nuevas tecnologías, el aumento del precio medio del libro, de distribuidoras, publicaciones y el creciente número de editoriales, entre otros. Cada una de las editoriales entrevistadas ha enfrentado esos cambios con diferentes estrategias. La editorial Balduque, la más pequeña de las entrevistadas, surgió durante la crisis 
económica y orientó sus estrategias de comunicación hacia el uso de plataformas digitales para ganar visibilidad y mantener las relaciones con los lectores, además de cuidar su imagen de marca. La editorial Alba, mediana, ha reaccionado ante estos cambios encaminando sus estrategias hacia el ahorro económico, mediante la reducción de ejemplares destinados a promoción y la segmentación del público al que dirigirse, según los intereses de los lectores. La editorial Anagrama, la más grande de las tres, ha afrontado esos cambios adaptándose de manera progresiva a la utilización de las nuevas plataformas digitales y apostando por el e-book. La editorial Balduque no cuenta con departamento de comunicación; mientras que la editorial Alba utiliza los términos "marketing" y "comunicación" de forma indistinta, al igual que la editorial Anagrama, pero esta última sí cuenta con un departamento de comunicación.

Aquí se presenta un primer cuadro con las principales estrategias de comunicación que llevan a cabo cada una de las editoriales durante el proceso de promoción de las publicaciones.

\begin{tabular}{|c|c|c|c|}
\hline $\begin{array}{l}\text { Estrategias de } \\
\text { comunicación }\end{array}$ & Balduque & Alba & Anagrama \\
\hline $\begin{array}{l}\text { Promoción antes } \\
\text { de la publicación } \\
\text { del libro }\end{array}$ & $\begin{array}{l}\text { - Redes sociales (Facebook y } \\
\text { Twitter): campañas teaser } \\
\text { (anticipadas o de avance) y } \\
\text { noticias. } \\
\text { - Uso más habitual de } \\
\text { Facebook para crear } \\
\text { comunidades. } \\
\text { - Web y boletín informativo. } \\
\text { - Preventas. } \\
\text { - Envío de reseñas a críticos y } \\
\text { periódicos. }\end{array}$ & $\begin{array}{l}\text { - } \text { Preventas online. } \\
\text { - } \text { Entrevistas a autores } \\
\text { - } \text { Redes sociales y web } \\
\text { para informar de las } \\
\text { novedades. }\end{array}$ & $\begin{array}{l}\text { - Promoción del } \\
\text { libro cuando sale } \\
\text { a la venta. } \\
\text { - Envío de galera- } \\
\text { das y ejemplares } \\
\text { a la prensa y } \\
\text { críticos. } \\
\text { - Información } \\
\text { sobre novedades } \\
\text { a distribuidores y } \\
\text { librerías. }\end{array}$ \\
\hline $\begin{array}{l}\text { Promoción durante } \\
\text { la publicación del } \\
\text { libro }\end{array}$ & $\begin{array}{l}\text { - Acciones convencionales: } \\
\text { presentaciones, entrevistas, } \\
\text { ruedas de prensa, etcétera. } \\
\text { - Marketing word of mouth. }\end{array}$ & $\begin{array}{l}\text { Acciones conven- } \\
\text { cionales: presenta- } \\
\text { ciones, entrevistas, } \\
\text { ruedas de prensa, } \\
\text { etcétera. }\end{array}$ & $\begin{array}{l}\text { - Acciones } \\
\text { convencionales: } \\
\text { presentaciones, } \\
\text { entrevistas, rue- } \\
\text { das de prensa, } \\
\text { etcétera. } \\
\text { - Facebooky } \\
\text { Twitter Ads. } \\
\text { - Booktrailers. }\end{array}$ \\
\hline
\end{tabular}




\begin{tabular}{|c|c|c|c|}
\hline $\begin{array}{l}\text { Seguimiento de la } \\
\text { campaña }\end{array}$ & $\begin{array}{l}\text { - Impacto en prensa y en } \\
\text { redes sociales. } \\
\text { - Comprobación de ventas } \\
\text { mediante liquidaciones. } \\
\text { - Cambios en plena campaña } \\
\text { si la promoción no funciona: } \\
\text { más presentaciones, } \\
\text { entrevistas, etcétera. }\end{array}$ & $\begin{array}{l}\text { - Impacto en prensa y } \\
\text { en redes sociales. } \\
\text { - No realizan cambios } \\
\text { durante la campaña } \\
\text { salvo el envío de algún } \\
\text { ejemplar y nota de } \\
\text { prensa. }\end{array}$ & $\begin{array}{l}\text { - Análisis de } \\
\text { los fallos de la } \\
\text { comunicación } \\
\text { - Entrevistas y } \\
\text { Newsletter. }\end{array}$ \\
\hline $\begin{array}{l}\text { Promoción después } \\
\text { de la publicación } \\
\text { del libro }\end{array}$ & $\begin{array}{l}\text { - Promoción sólo en el caso } \\
\text { de que el libro sea noticia } \\
\text { por tratar un tema social } \\
\text { actual o tenga adaptación } \\
\text { cinematográfica. }\end{array}$ & $\begin{array}{l}\text { - Promoción sólo en el } \\
\text { caso de que el libro } \\
\text { sea noticia por tratar } \\
\text { un tema social actual } \\
\text { tenga adaptación } \\
\text { cinematográfica. }\end{array}$ & $\begin{array}{l}\text { - No realiza } \\
\text { acciones tras } \\
\text { el proceso de } \\
\text { promoción. }\end{array}$ \\
\hline
\end{tabular}

Cuadro 1. Estrategias de comunicación durante el proceso de promoción editorial.

Fuente: elaboración propia.

Finalmente, se muestra un segundo cuadro, con un análisis de las debilidades, fortalezas y oportunidades de las editoriales, respecto del proceso de promoción de los libros y los cambios que se producen en su entorno.

\begin{tabular}{|c|c|c|c|}
\hline Análisis & Balduque & Alba & Anagrama \\
\hline Debilidades & $\begin{array}{l}\text { - Falta de presupuesto } \\
\text { para publicidad. } \\
\text { - Necesidad de actuali- } \\
\text { zación web. } \\
\text { - Falta de tiempo para } \\
\text { los autores. }\end{array}$ & $\begin{array}{l}\text { - Envío masivo de } \\
\text { ejemplares. }\end{array}$ & $\begin{array}{l}\text { - Envejecimiento de } \\
\text { su público objetivo. }\end{array}$ \\
\hline Fortalezas & $\begin{array}{l}\text { - Imagen de marca. } \\
\text { - Profesionalidad y con- } \\
\text { secución de objetivos. }\end{array}$ & $\begin{array}{l}\text { - Especialización en } \\
\text { obras clásicas. } \\
\text { - Calidad de las } \\
\text { traducciones. }\end{array}$ & $\begin{array}{l}\text { - Precio asequible } \\
\text { de sus libros de } \\
\text { bolsillo. } \\
\text { - Cuidado en el } \\
\text { diseño. }\end{array}$ \\
\hline Oportunidades & $\begin{array}{l}\text { - Nuevas tecnologías } \\
\text { - Impresión bajo } \\
\text { demanda. } \\
\text { - Posibilidad de negocio } \\
\text { con distribuidores. } \\
\text { - Autores de prestigio en } \\
\text { editoriales pequeñas. }\end{array}$ & - Nuevas tecnologías. & - Nuevas tecnologías \\
\hline
\end{tabular}

Cuadro 2. Análisis FODA de las editoriales sobre el proceso de promoción editorial y los cambios en su entorno.

Fuente: elaboración propia. 


\section{Conclusiones}

En cuanto a las acciones de venta personal en librerías, las tres editoriales cuentan con distribuidores o redes comerciales encargadas de las relaciones con los puntos de distribución; las editoriales no les dan directrices concretas ni dejan constancia de que los libreros realicen algún tipo de formación en marketing o comunicación.

La publicidad, en general, no es un medio habitual utilizado por las editoriales, dado su alto coste económico, salvo la que realizan en prensa, para críticos literarios y la publicidad en el punto de venta.

Asimismo, las acciones de promoción de ventas que realizan están destinadas a la preventa del libro. Ninguna de las tres editoriales deja constancia de realizar actividades de patrocinio, aunque sí colaboran en programas culturales.

Las acciones de relaciones públicas y publicidad que realizan las tres editoriales tienen la misma base, pero con distintas especificaciones. La editorial Balduque busca crear un clima de confianza con los lectores para lograr la fidelización a través de las redes sociales, especialmente en Facebook, por su facilidad para crear comunidades y transmitir cercanía. En cambio, la editorial Alba mantiene la relación con sus lectores a través de talleres, según el tipo de publicación; en tanto que la editorial Anagrama realiza concursos en redes sociales para los lectores. El trato con el autor de las tres editoriales se basa en las mismas acciones, por ejemplo, la creación de entrevistas, actos públicos, presentaciones, etc. Además de utilizar las mismas acciones de comunicación con la prensa y los críticos, como envíos de galeradas y ejemplares, las notas de prensa, ruedas de prensa, entre otras.

El uso de las nuevas tecnologías forma parte de las estrategias de comunicación de las tres editoriales. Alba sólo especifica el uso de las redes sociales Facebook, Twitter e Instagram y su propia web como medio de información de las novedades; mientras que Balduque utiliza Facebook con asiduidad para informar de las novedades editoriales, y Twitter en menor medida, además de que dispone también de una página web propia. No obstante, ninguna de las dos editoriales especifica utilizar estas plataformas para lanzar campañas de comunicación con una estrategia definida, ni usa las herramientas de las que disponen para crear anuncios. Ambas utilizan el correo electrónico comercial como herramienta para mantener informados a sus suscriptores de las novedades editoriales a través del envío de boletines informativos (newsletter). Anagrama también utiliza estas mismas herramientas, pero también genera campañas publicitarias a través de Facebook y Twitter Ads. Otra forma de adaptarse a las nuevas tecnologías es su apuesta por el libro electrónico. 
Las nuevas tecnologías y plataformas digitales aún se utilizan de forma muy analógica en estas editoriales. En general, no se llevan a cabo campañas de comunicación definidas y apenas se saca partido de todo lo que estas plataformas pueden ofrecer.

Cabe señalar que existe una confusión respecto de los conceptos de marketing y comunicación. Las editoriales independientes han surgido durante la crisis económica, lo que ha conllevado un mejor aprovechamiento de los cambios que ha habido, como una adaptación al uso de plataformas digitales, negocios con distribuidoras que antes sólo trabajaban con editoriales grandes y más espacio en las librerías para las editoriales pequeñas, entre otras.

También ha quedado definido el carácter de aleatoriedad de una editorial pequeña, que es más propensa a las acciones que surgen a corto plazo, debido a que su objetivo inmediato es el de conseguir la rentabilidad. En cambio, una editorial más grande, con objetivos de crecimiento, cuenta con departamento propio de comunicación. Sin embargo, la crisis económica ha afectado considerablemente a las grandes editoriales, las cuales han tenido que orientar sus estrategias hacia el ahorro económico y su proceso de adaptación a las nuevas tecnologías es más lento, dada la larga trayectoria de la editorial antes de su implantación.

En general, se aprecia la necesidad de una formación en mercadotecnia y comunicación para el personal responsable, y también como forma de adaptarse a los medios digitales, tanto por parte de las editoriales como del personal de ventas. La confusión en el uso de los términos demanda una mejor definición y una implantación más rigurosa de los planes de comunicación.

\section{REFERENCIAS}

Cole, David. 2003. Marketing editorial: la guía. Trad. de Gabriela Ubaldini. México: Conaculta.

Escribano Hernández, Asunción. 2012. La retórica publicitaria editorial: el arte de vender un libro. Madrid: Arco/Libros.

Gómez-Tarragona, Daniel. 2010. Marketing editorial: cómo satisfacer las necesidades de los lectores de libros. Madrid: Grupo Anaya.

Ministerio de Educación, Cultura y Deporte. 2018. "El sector del libro en España 2016”, en <https:/www.mecd.gob.es/dam/jcr:08c8edc0-2753-4306-9624-732c63843df0/ sector-libro-2018.pdf $>$.

Ministerio de Educación, Cultura y Deporte. 2017. "El sector del libro en España 2015”, en <https://www.mecd.gob.es/dam/jcr:bd29177e-2c26-4dbf-80d5-cc40a12a676d/ el-sector-del-libro-en-espa-a--junio-2017.pdf>. 
Ministerio de Educación, Cultura y Deporte. 2016. "El sector del libro en España 2013-2015”, en <http://www.mecd.gob.es/dam/jcr:f6104e45-f435-408a-a144-d7d73d3bdb5f/el-sector-del-libro-en-espa-a---enero-2016.pdf $>$.

Ministerio de Educación, Cultura y Deporte. 2015. "El sector del libro en España 2013-2015”, en <http:/www.mecd.gob.es/dam/jcr:d97a2217-70d0-4a72-b38604c3ae1ee9c2/sector-libro-abril2015.pdf $>$.

\section{Para citar este texto:}

Gómez López, Almudena y Pedro Antonio Hellín Ortuño. 2019. "Estrategias de comunicación de las editoriales literarias de España”. Investigación Bibliotecológica: archivonomía, bibliotecología e información 33 (80): 57-71.

http://dx.doi.org/10.22201/iibi.24488321xe.2019.80.57982 
